Research Round Up-Statin Prescribing, Preventative Use and Lipid Levels

Introduction

The last research round up provided you with an overview of some papers related to polypharmacy and multimorbidity and the impact the COVID-19 global pandemic has had on prescribing practices in these areas as well as the risk polypharmacy and multimorbidity carry to those who contract COVID-19. This month we will be reviewing papers relating to prescribing of statins and lipid modification goals in the management of hyperlipidaemia and prevention of cardiovascular disease.

Current Perspectives on the Attainment of Lipid Modification Goals Relating to the Use of Statins and Ezetimibe for the Prevention of Cardiovascular Disease in the United Kingdom

This review of the literature published in the Journal of Vascular Health \& Risk Management, looked at the important area of the use of the drug group statins and the ezetimibe (a cholesterol absorption inhibitor) and the link to achievement of lipid modification goals and their use in prevention of cardiovascular disease. This UK based review was conducted between January 2017 and February 2020. The authors give a good introduction to cholesterol and its role in cardiovascular disease and outline the benefits of modifying lipid levels as a strategy to reduced risk of development of cardiovascular disease. They provide good evidence to support their statement that

'Despite widespread evidence of the effectiveness of lipid modification for the reduction of cardiovascular disease (CVD) risk, lipid modification goals are commonly underachieved in the United Kingdom (UK)'.

Identified publications were reviewed against key clinical guidelines for lipid management in relation to CVD risk from the National Institute for Health and Care Excellence (NICE, CG181), the Scottish Intercollegiate Guidelines Network (SIGN, 149) and European Society of Cardiology (ESC)/European Atherosclerosis Society (EAS). The aim was to identify if reporting in the literature could evidence that cholesterol lowering goals were achieved in clinical practice in line with the most current guidance. Current guidance by NICE and SIGN specifies that treatment should achieve a greater than $40 \%$ reduction in non-high-density lipoprotein cholesterol (non-HDLC) at 3 months of treatment, while the ESC/EAS place emphasis on the lowering of low density lipoprotein (LDL-C) and total cholesterol.

They found from the literature that was critically reviewed over that 3 year period, that despite widespread availability of guidance and consistent messaging that lipid lowering goals should be ambitious, current evidence suggests a significant proportion of UK patients have sub-optimal reductions in cholesterol/non-HDL-C/LDL-C. The reasons for this that were reported in the reviewed literature were not simple. Those reported included lack of compliance with the guidelines by prescribers (and this was found to be truer with regard to high-intensity statin prescribing), along with lack of adherence by patients when prescribing occurred. The reasons for patient nonadherence could be related to statin intolerance and reluctance with a hint at wider genetic factors being implicated.

A number of possible strategies to improve current lipid management and attainment of lipidlowering goals were identified, including improving the patient-healthcare professional partnership, conducting audits of local prescribing versus guidance, implementing plans for the refinement of current services and considering alternative options such as cost-effective single pill combinations for improving adherence. They conclude that additional options are required to manage and treat 
patients at high risk of CVD events, where lipid lowering goals are not attained with statins and ezetimibe alone.

https://www.ncbi.nlm.nih.gov/pmc/articles/PMC8149323/pdf/vhrm-17-227.pdf

Reynolds, T.M., Pottle, A. \& Quoraishi.S.H. (2021) Current Perspectives on the Attainment of Lipid Modification Goals Relating to the Use of Statins and Ezetimibe for the Prevention of Cardiovascular Disease in the United Kingdom Vascular Health \& Risk Management :17 227-237

\section{Factors predicting statin prescribing for primary prevention: a historical cohort study}

This historical cohort study, published in the British Journal of General Practice aimed to investigate the factors that influence the prescribing of statins in response to lipid level testing using data from between 2012 and 2016 obtained from an anonymised database of primary care records for practices in England and Wales contributing to IQVIA Medical Research Data (IMRD)-UK (formally The Health Improvement Network). IMRD contains routine patient data from $>500$ general practices and is generalisable to the UK population. The cohort comprised 686560 entries (lipid test results) between 2012 and 2016 from 383416 statin-naive patients without previous Cardiovascular Disease (CVD). Coded QRISK2 scores (a measure of CVD risk) were extracted, with variables used in calculating QRISK2 and factors that might influence statin prescribing. If a QRISK2 score was not coded, it was calculated post hoc. The outcome of interest was if there was initiation of a statin within 60 days of the lipid test result.

The authors provide a good background and overview of the use of statins particularly linking this to primary prevention of CVD. They state that despite evidence supporting their use, the literature suggested that statin use was sub optimal and this prompted their study. The results of the study showed that of the entries, $146693(21.4 \%)$ had a coded QRISK2 score. Statins were initiated in 6.6\% (95\% confidence interval $[\mathrm{Cl}]=6.4 \%$ to $6.7 \%)$ of those with coded and $4.1 \%(95 \% \mathrm{Cl}=4.0 \%$ to $4.1 \%)$ of uncoded QRISK2 $(P<0.001)$. Statin initiations were consistent with National Institute for Health and Care Excellence guideline recommendations in $85.0 \%(95 \% \mathrm{Cl}=84.2 \%$ to $85.8 \%)$ of coded and $44.2 \%(95 \% \mathrm{Cl}=43.5 \%$ to $44.9 \%)$ of uncoded QRISK2 groups $(P<0.001)$. When coded, QRISK2 score was the main predictor of statin initiation, but total cholesterol was the main predictor when a QRISK2 score was not coded.

The authors conclude from this that when a QRISK2 score is coded, prescribing is more consistent with guidelines. With no QRISK2 score, prescribing is mainly based on total cholesterol. Using QRISK2 is associated with statin prescribing that is more likely to benefit patients. Promoting the routine CVD risk estimation is essential to optimise decision making. They suggest that further research is necessary to investigate why such a significant proportion of statin prescribing is still done based on cholesterol levels rather than using the QRISK2 calculation. They also suggest that an action which could increase the number of primary care prescribers who measure QRISK2 could improve adherence to guidelines for prescribing statins.

\section{https://bjgp.org/content/bjgp/71/704/e219.full.pdf}

Finnikin, S., Willis, B.H., Ryan, R., Evans, T. \& Marshall, T. (2021) Factors predicting statin prescribing for primary prevention: a historical cohort study British Journal of General Practice: e219 
Lipid levels and major adverse cardiovascular events in patients initiated on statins for primary prevention: an international population-based cohort study protocol

This international population-based cohort study, published in the British Journal of General Practice (open), aimed to assess whether lower levels of cholesterol were reliably associated with a lower risk of cardiovascular disease (CVD) specifically relating to primary prevention. The authors describe the proposed study protocol and give a good background to their rationale and the need for this work. The 3-site study intends to extract data from electronic databases in the UK, New Zealand and Hong Kong. These databases are 'The Clinical Practice Research Datalink (CPRD) GOLD' (UK) and 'PREDICT-CVD' (New Zealand) which are primary care databases; AND 'the Clinical Data and Analysis Reporting System (CDARS)' (Hong Kong) which is an integrated health system database (primary and hospital care). The data the authors intend to extract are outlined as follows

- New statin users without a history of atherosclerotic CVD, heart failure, or chronic kidney disease, with baseline and follow-up lipid levels will be eligible for inclusion.

- Patients will be classified according to LDL-C $(<1.4,1.4-1.7,1.8-2.5$, and $\geq 2.6 \mathrm{mmol} / \mathrm{l})$ and non-HDL-C $(<2.2,2.2-2.5,2.6-3.3$, and $\geq 3.4 \mathrm{mmol} / \mathrm{l})$ concentrations 24 months after initiating statin therapy.

The main outcome measure the researchers are looking for is any Major Adverse Cardiovascular Event (MACE). They define these as Coronary Heart Disease, Stroke or death due to a cardiovascular event. There are secondary outcomes of interest including all-cause mortality and the individual components of MACE. Sensitivity levels will be considered by measurement of circulating lipid levels with bloods taken at 3 and 12 months after commencement of a statin.

The researchers hope that their results will assist and inform clinicians to strive for lipid level goals and to adhere to evidence based clinical guidelines with regard to statin prescribing for CVD prevention. They hope this will address the variability in prescribing practice around primary prevention.

https://bigpopen.org/content/bigpoa/5/1/bigpopen20X101127.full.pdf

Blais, J.E., Akyea, R.K., Coetzee, A., Chan, A.H.Y., Lau, W.C.Y., Man, K.K.C., Harrison, J., Chan, E.W., Beyene, K.A., Wong, I.C.K. \& Weng, S. (2020)_Lipid levels and major adverse cardiovascular events in patients initiated on statins for primary prevention: an international population-based cohort study protocol BJGP Open.

\section{Conclusion}

Statins as a group of lipid lowering medications are widely used in the UK to lower lipid levels and in primary and secondary prevention of cardiovascular disease. Robust prescribing guidelines are available, but studies show that adherence to these guidelines is not universally achieved. Clearly more research is required in this area primarily to ascertain the reasons for this but also to provided guidance and information to assist prescribers in their decision making in this important area of practice. 